\title{
The Stochastic Gravitational Wave Background Generated by Cosmic String Networks
}

\author{
L. Sousa ${ }^{1}$ and P. P. Avelino ${ }^{1} 2$ \\ ${ }^{1}$ Centro de Astrofísica da Universidade do Porto, Rua das Estrelas, 4150-762 Porto, Portugal \\ ${ }^{2}$ Departamento de Física e Astronomia da Faculdade de Ciências da Universidade do Porto, \\ Rua do Campo Alegre 687, 4169-007 Porto, Portugal \\ emails: Lara.Sousa@astro.up.pt, pedro.avelino@astro.up.pt
}

\begin{abstract}
Cosmic string interactions often result in the formation of cosmic string loops that detach from the long string network and radiate their energy in the form of gravitational waves. Loop production occurs copiously throughout the cosmological evolution of a cosmic string network and the superimposition of their emissions gives rise to a stochastic gravitational In this essay, we briefly review our recent work on the stochastic gravitational wave background generated by cosmic string networks and introduce a set of numerical and analytical tools for the computation of this background.
\end{abstract}

Keywords. cosmology: theory, early universe, gravitational waves

\section{Introduction}

The production of cosmic string networks as remnants of symmetry-breaking phase transitions in the early universe is predicted in a wide variety of grand unified scenarios. These cosmic string networks, despite being formed in the early universe, may survive throughout the cosmological history, potentially leaving behind a plethora of observational signatures. One such signature would be a stochastic gravitational wave background (SGWB) generated as a result of the radiative emissions of cosmic string loops (Vilenkin (1981)). These loops are created in significant numbers as a result of cosmic string collisions and interactions (for instance, when a string self-intersects or when two strings intersect simultaneously at more than one point). Once these loops are created, they detach from the long string network and start to oscillate relativistically under the effect of their tension and decay radiatively by emitting gravitational waves.

If one assumes that the cosmic string network is statistically homogeneous on sufficiently large scales, two variables are sufficient to describe its large-scale dynamics: the characteristic lengthscale $-L \equiv(\mu / \rho)^{1 / 2}$, where $\mu$ is the string tension, and $\rho$ is the average energy density of long strings - and its root-mean-square velocity, $\bar{v}$. Under this assumption, the rate of loop production (per comoving volume) is given by

$$
\frac{d n_{c}}{d t}=\frac{\tilde{c}}{\alpha} \frac{\bar{v}}{L^{4}}
$$

where $\alpha$ is a parameter that characterizes the size of loops, $l_{c}=\alpha L\left(t_{c}\right)$, at the moment of creation, $t_{c}$, and $\tilde{c}$ is a phenomenological parameter that characterises the efficiency of the loop-chopping mechanism. The rate of loop production is, then, strongly dependent on the large-scale properties of the network and, therefore, its accurate characterisation requires an accurate description of cosmic string network dynamics. 

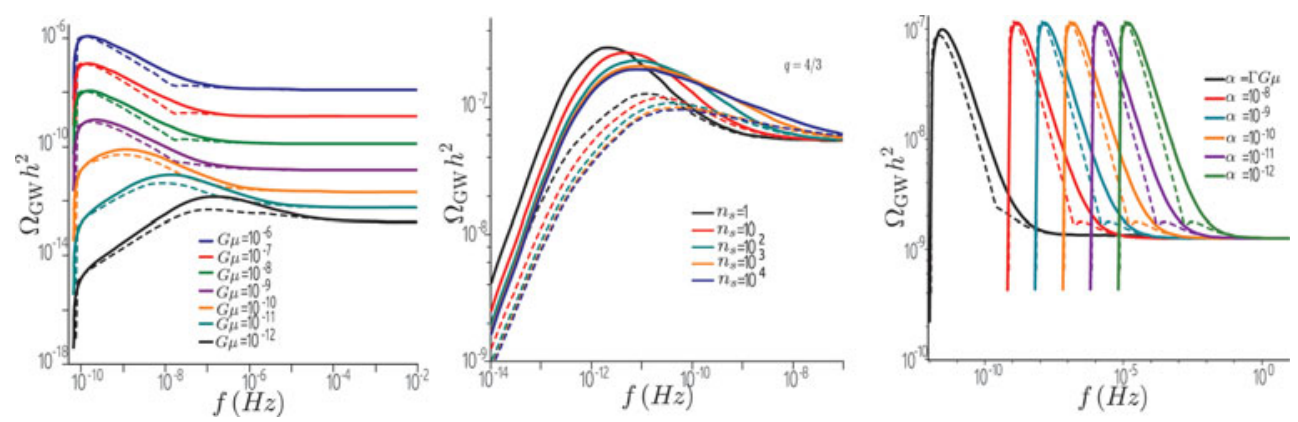

Figure 1. Same examples of SGWB power spectra. From left to right: the SGWB spectrum for 1. various values of cosmic string tension (and fixed $\alpha=10^{-7}$ ); 2. large cuspy loops $\left(\alpha=10^{-1}\right.$ ), considering $n_{s}$ harmonic modes of emission; 3. small loops (and fixed $G \mu=10^{-7}$ ). Solid lines represent the SGWB spectra computed numerically using the VOS model to describe cosmic string dynamics, while dashed lines represent the spectra of networks experiencing scale-invariant evolution.

\section{SGWB: Numerical results}

The Stochastic gravitational wave background is often quantified using the spectral density of gravitational waves per logarithmic frequency $(f)$ interval, in units of critical density $\left(\rho_{\mathrm{c}}\right)$,

$$
\Omega_{\mathrm{GW}}=\frac{1}{\rho_{\mathrm{c}}} \frac{d \rho_{\mathrm{GW}}}{d \log f},
$$

where $\rho_{\mathrm{GW}}$ is the gravitational radiation energy density. This quantity is strongly dependent on the rate of loop production as a function of time, and, consequently, on the largescale cosmic string parameters. In this context, it is generally assumed that cosmic string networks experience scale-invariant evolution throughout cosmological history, with the characteristic lengthscale growing linearly with time $(L \propto t)$ and constant $\bar{v}$. However, this is not an accurate depiction of realistic cosmic string network dynamics: once the radiation-matter transition is triggered, the network cannot maintain scale-invariant evolution. In order to improve current estimations of SGWB spectrum, we have developed, in Sousa \& Avelino (2013), an algorithm to compute the spectral density of gravitational waves generated by realistic cosmic string networks. This algorithm relaxes the assumption of scale-invariant cosmic string evolution, and uses the Velocity-Dependent One-Scale (VOS) model (Martins \& Shellard (1996)) — which was previously shown to provide an accurate description of the large-scale dynamics of cosmic string networks to describe their dynamics.

We have demonstrated that the simplifying assumptions about network dynamics used in the majority of SGWB computations lead to an underestimation of the number of loops created during most of the matter era and that this causes a significant underestimation of the amplitude and broadness of the peak of the spectrum (see Fig. 1). As a result, the total gravitational wave energy density at the present time obtained using the VOS model may be $25-70 \%$ larger (depending on the size of cosmic string loops) than that obtained under the scale-invariant assumption. The use of simplified models of string evolution may, therefore, lead to inaccuracies in the computation of the observational constraints on cosmic string tension using data from direct or indirect gravitational wave detection experiments. 

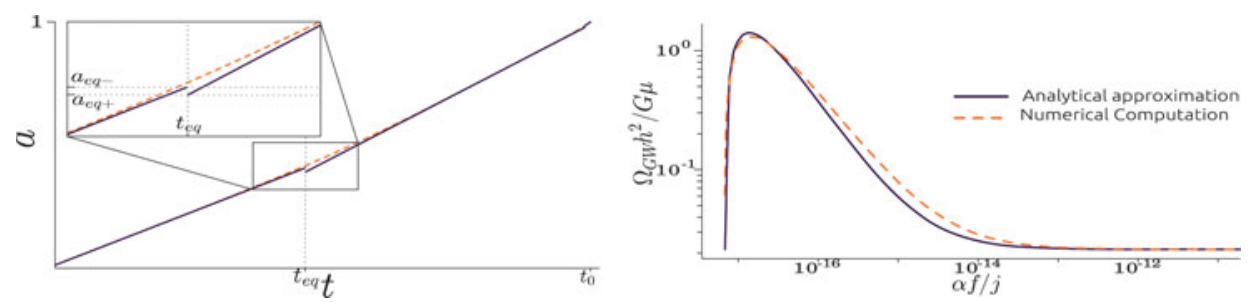

Figure 2. Left Panel: Fitting function for the cosmological scale factor. Right Panel: Analytical approximation to the SGWB power spectrum in the small loop regime.

\section{SGWB: Analytical Approximation for the small-loop regime}

If loops are small — smaller than the gravitational backreaction scale — they have a short lifespan, surviving less than a Hubble time. In this limit, it is reasonable to assume that loops radiate their energy effectively immediately in the cosmological timescale. Using this simplification, we have developed in Sousa \& Avelino (2014) an alternative method to compute the SGWB spectrum in the small-loop regime that has the advantage of requiring significantly less computation time.

This method also allowed us to derive an analytical approximation to the realistic SGWB generated by small cosmic string loops:

$$
\Omega_{\mathrm{gw}}^{j} h^{2}(f)=\mathcal{K}\left\{\frac{\bar{v}_{r}}{\xi_{r}^{3}} \frac{\alpha}{j t_{e q}^{2}}\left(\frac{a_{e q-}}{a_{0}}\right)^{4}+\frac{3}{f} \frac{\bar{v}_{m}}{\xi_{m}^{4} t_{\Lambda}^{3}}\left(\frac{a_{\Lambda-}}{a_{0}}\right)^{5}\left[1-\frac{2 j}{\alpha \xi_{m} t_{\Lambda} f}\left(\frac{a_{\Lambda-}}{a_{0}}\right)\right]\right\},
$$

for $f \geqslant 2 j a_{\Lambda-} / t_{\Lambda}$. Here we have introduced the constants $\mathcal{K}=\left(16 j \pi G \mu h^{2} \tilde{c}\right) /\left(3 H_{0}^{2} \alpha\right)$, and $a_{\Lambda-}=a_{e q+}\left(t_{\Lambda} / t_{e q}\right)^{2 / 3}$, used $j=1,2, \ldots$ to label the harmonic mode of loop emission, and $t_{\mathrm{eq}}$ and $t_{\Lambda}$, respectively, for the time of radiation-matter equality and of mattercosmological-constant equality. Moreover, $a_{0}$ and $H_{0}$ represent, respectively, the value of the scale factor and the Hubble parameter at the present time. In deriving this expression, we have assumed that the network undergoes scale-invariant evolution throughout cosmological history - characterized by $\bar{v}=\bar{v}_{r}\left(\bar{v}=\bar{v}_{m}\right)$ and $L=\xi_{r} t\left(L=\xi_{m} t\right)$ during the radiation (matter) era. We have also assumed that the dynamics of the universe is determined, at any given time, by the dominant component of the its energy density, in order to construct an analytical fitting function for the scale factor (see the left panel of Fig. 2). This analytical approximation to the SGWB spectrum provides an excellent fit to that of realistic cosmic string networks (see the right panel of Fig. 2). It constitutes a useful tool for a first estimation of the SGWB spectrum, thus allowing for simple estimates of the associated observational constraints in the small-loop regime.

\section{Acknowledgements}

L.S. is supported by FCT and POPH/FSE through the grant SFRH/BPD/76324/2011. P.P.A. is supported by FCT and POPH/FSE through contract IF/00863/2012. This work was also partially supported by grant PTDC/FIS/111725/2009 (FCT).

\section{References}

Vilenkin, A. 1981, Phys.Lett. B107, 47

Sousa, L. \& Avelino, P. P. 2013, Phys.Rev. 2 D88, 023516

Sousa, L. \& Avelino, P. P. 2014, Phys.Rev. D89, 083503

Martins, C. J. A. P. \& Shellard, E. P. S 1996, Phys.Rev. D54, 2535-2556 